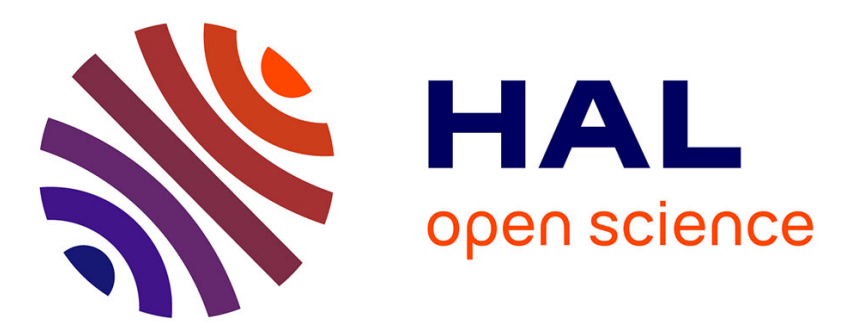

\title{
Experimental evaluation of transverse friction between fibers
}

Houssem Eddine Gassara, Gérald Barbier, Christiane Wagner Kocher, Artan Sinoimeri, Besnik Pumo

\section{- To cite this version:}

Houssem Eddine Gassara, Gérald Barbier, Christiane Wagner Kocher, Artan Sinoimeri, Besnik Pumo. Experimental evaluation of transverse friction between fibers. Tribology International, 2018, 119, pp.112 - 122. 10.1016/j.triboint.2017.10.035 . hal-01668780

\section{HAL Id: hal-01668780 \\ https://hal.science/hal-01668780}

Submitted on 20 Dec 2017

HAL is a multi-disciplinary open access archive for the deposit and dissemination of scientific research documents, whether they are published or not. The documents may come from teaching and research institutions in France or abroad, or from public or private research centers.
L'archive ouverte pluridisciplinaire HAL, est destinée au dépôt et à la diffusion de documents scientifiques de niveau recherche, publiés ou non, émanant des établissements d'enseignement et de recherche français ou étrangers, des laboratoires publics ou privés. 


\section{EXPERIMENTAL EVALUATION OF TRANSVERSAL FRICTION BETWEEN FIBERS}

Houssem Eddine Gassara ${ }^{a}$, Gérald Barbier ${ }^{\mathrm{a}}$, Christiane Wagner Kocher ${ }^{\mathrm{a}, \mathrm{b}}$, Artan Sinoimeri ${ }^{\mathrm{a},{ }^{*} \text {, }}$ Besnik Pumo ${ }^{c}$

a LPMT - Laboratoire de Physique et Mécanique Textiles, Ecole Nationale Supérieure d'Ingénieurs Sud-Alsace, Université de Haute-Alsace, 11 rue Alfred Werner, 68093 Mulhouse, France

${ }^{\mathrm{b}}$ LMGC - Laboratoire de Mécanique et Génie Civil, Univ. Montpellier, CNRS, 860 Rue de St - Priest, 34095 Montpellier, France

' IRHS, Agrocampus Ouest, INRA, Université d'Angers, SFR QuaSaV, 49071 Beaucouzé, France

\section{ABSTRACT}

The mechanical behavior of fibrous assemblies, particularly the transverse behavior of fiber yarns and multifilament strands depends, inter alia, on the fiber-to-fiber contact and sliding. All studies on inter fiber friction presented in the literature focus on cases where the relative inter fiber motion occurs either in longitudinal-to-longitudinal or longitudinal-to-transverse direction. The transverse-totransverse inter fiber friction plays an important role as far as the mechanical transverse behavior of fiber yarns and multifilament strands are concerned. In order to evaluate the case named here transverse friction, the present study proposes an original method and an associated model analyzing the transversal component of friction between two oblique crossing fibers. A relatively simple and original statistical approach has also been developed to evaluate the confidence interval of friction coefficient. The first results show a relative stability of the transverse friction. This coefficient is also significantly inferior to the longitudinal one.

Key Words: FRICTION, FIBER, YARN, EXPERIMENTAL, MODELING

\section{Introduction}

During the processing of fiber assemblies in a well-organized structure, either a yarn, a thin or a 3-dimensionnal fabric, the fiber characteristics play an important role in the properties of the final product. Depending on the fiber type - staple or filament one -, much research has been carried out to study the fiber length, distribution in length, fiber fineness, tensile characteristics, etc. Fiber surface properties, and especially their frictional ones, affect strongly the fiber-to-yarn or fabric transformation processes as well as the structure and mechanical properties of final products. Particularly, the yarn and/or fabric transverse compression behavior, which is a relatively important topic while numerically modeling the mechanical behavior of fibrous structures in 3-dimensionnal composite structures, depends strongly on the relative transverse fiber movement, and therefore on the inter fiber transverse friction. Despite the large number of fiber-to-fiber friction measuring devices, they focus on two main fiber configurations: violin string/bow configuration, which is often called 
orthogonal; and longitudinal-to-longitudinal configuration. The present study focuses on the transverse friction between individual fibers and proposes an original method to evaluate it.

\section{Background}

The pioneer in the friction study, Leonardo da Vinci (1452-1519) [1], suggested in 1508 that during the tangential movement between two bodies in contact, the tangential friction force is proportional to the normal force, and independent of the contact area.

Amontons (1663-1705) [2] formalized this relationship by introducing the friction coefficient $\mu$ as follows:

$$
F=\mu \cdot N
$$

where $F$ is the tangential frictional force and $N$ is the normal force between two bodies in contact and sliding over each other.

However, Amontons' law is not rigorously observed. Generally, there is an approximately linear relationship between the friction force and the force normal to the contact area, but that relationship varied from one material to another, from the static state to the dynamic one, and also depended on the relative movement speed.

Since 1950, the shear-adhesion theory of Bowden and Tabor [3] has proposed new methods in the study of friction and cohesion. According to this theory, the frictional force $F$ is generally related to the normal force $\mathrm{N}$ using the following equation:

$$
F=k \cdot N^{n}
$$

where $k$ and $n$ are the coefficients that characterize the friction, the index $n$ takes the type of deformation (plastic, elastic...) of the micro asperities of the two bodies in contact into account.

Let's now consider the fiber surfaces. Due to the spinning and drawing processes of manmade fibers, their surface characteristics are generally longitudinally oriented, as it can be seen from Figure 1. It follows that fiber-to-fiber or fiber-to-other body frictional behavior will depend strongly on the movement direction relative to the fiber axis. 

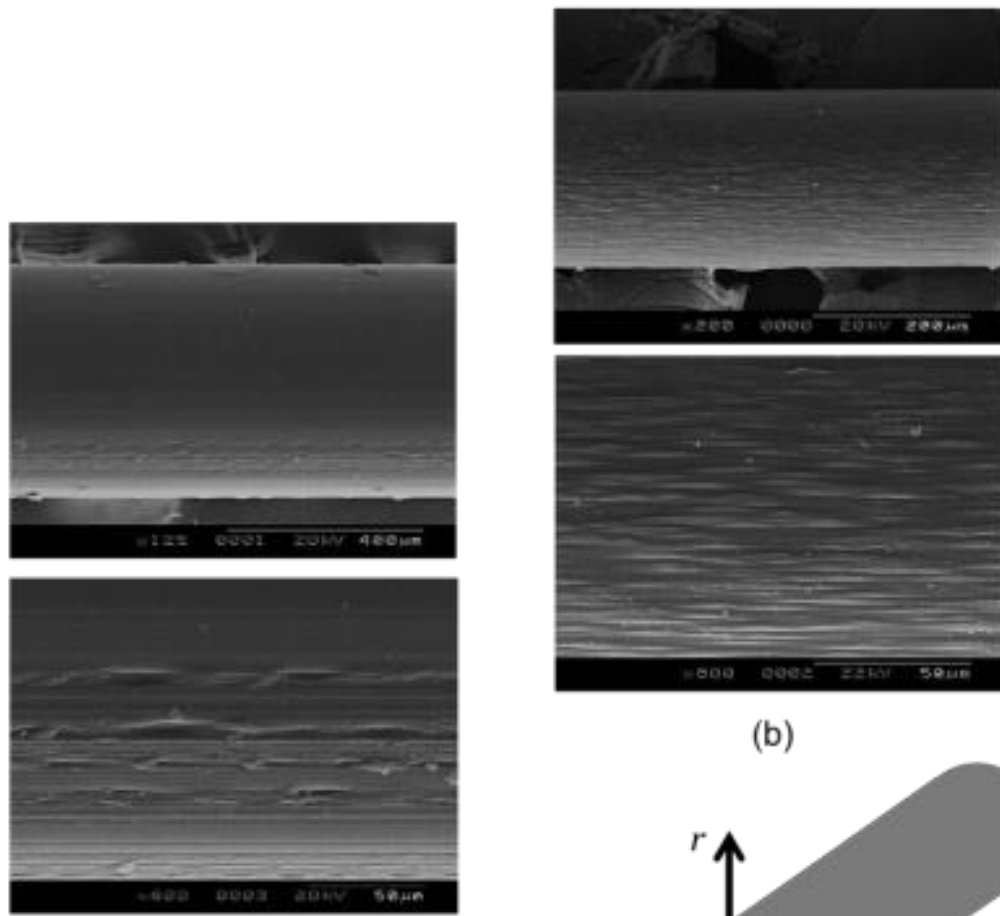

(a)

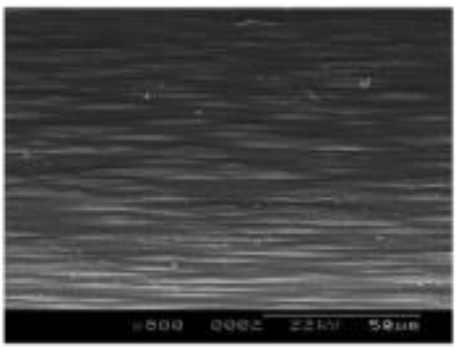

(b)

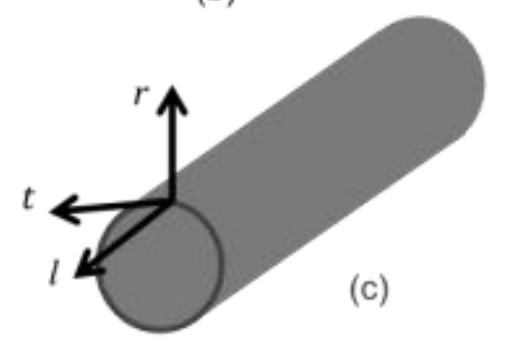

1)

Figure 1. SEM pictures of polyester (a) and polyamide 6-6 fibers (b),

(c) fiber longitudinal - $l$, transversal - $t$ and radial $-r$ directions

A lot of studies and measuring devices concerning fiber-to-fiber and fiber-to-other body friction can be found in the scientific literature. Despite the large number of those devices, as far as fiber-to-fiber friction is concerned, they can be classified in two main groups according to their measuring principles: longitudinal-to-transversal $l$ - $t$ friction type (case (a) in Figure 2) and longitudinal-to-longitudinal $l-l$ friction type (case (b) in Figure 2). Unfortunately, nothing has been proposed in the experimental literature on the transversal-to-transversal $t$ - $t$ friction measurement type (case (c) in Figure 2).

Howell [4] had developed the simplest device to characterize the $l-t$ friction. This device, as shown in Figure 2-a, was composed mainly of a frame B on which a first filament $E$ was horizontally mounted. This filament was brought into contact with a second filament $\mathrm{C}$, which was held tense from point $\mathrm{A}$ by a weight $\mathrm{W}$ fixed at its other end. Frame $\mathrm{B}$ was moved with filament $\mathrm{E}$ in the direction of the arrow, dragging filament $\mathrm{C}$, due to the frictional force, up to 
an equilibrium position where the frictional force was equilibrated by the tension of the suspended fiber. At that moment, slipping would occur. Distance $\mathrm{L}$ covered by fiber $\mathrm{C}$, creating an angle $\alpha$ measured between its two positions (see Figure 2a), permitted to determine the friction coefficient between the two fibers.

$$
\mu=\frac{w \cdot g \cdot \sin \alpha}{w \cdot g \cdot \sin \beta}=\frac{\sin \alpha}{\sin \beta}=\frac{L}{S}
$$

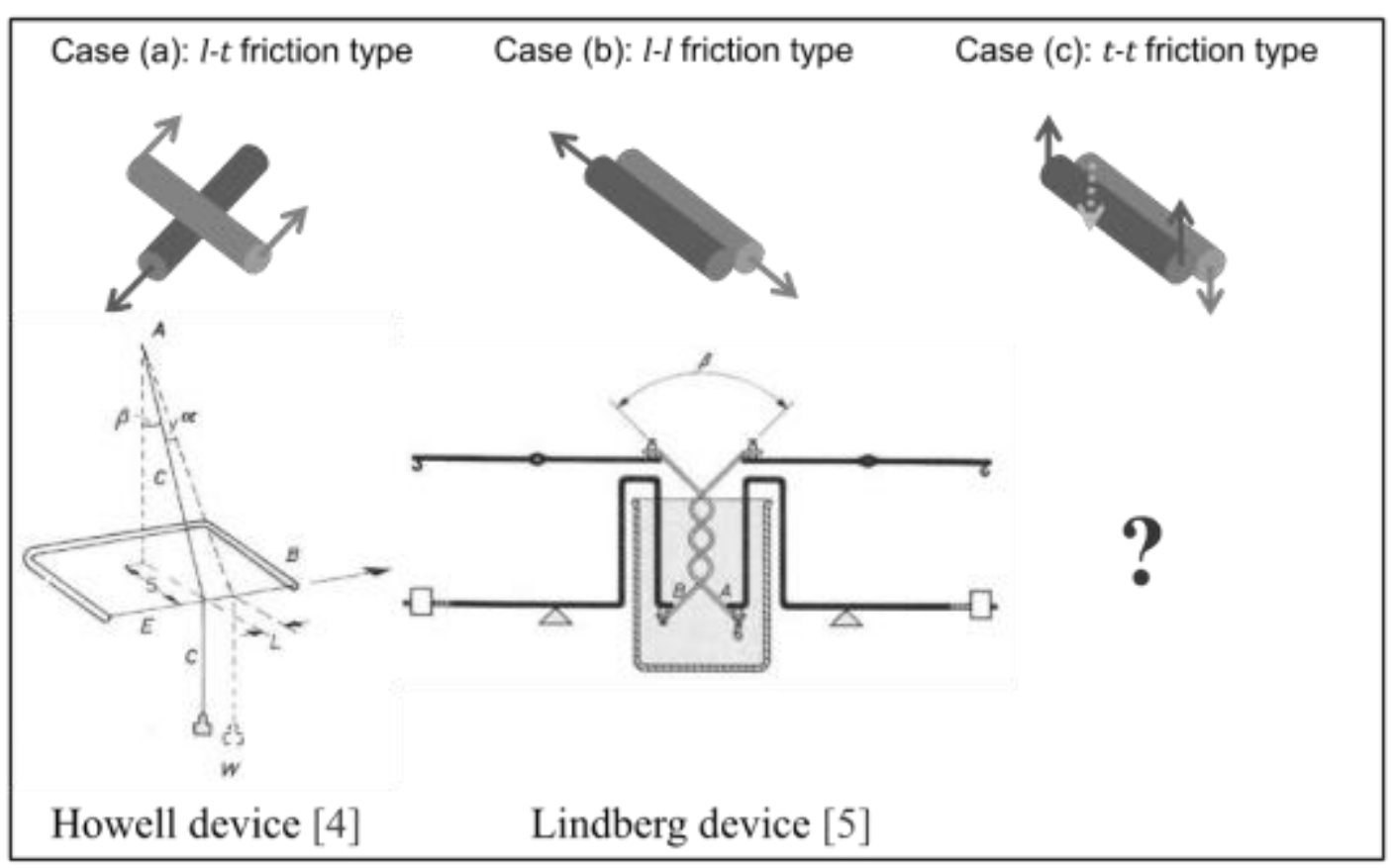

Figure 2. Frictional measurement in different configurations,

(a) Howell [4] and (b) Lindberg device [5]

The "twist" method to measure the inter-fiber friction was developed by Lindberg and Gralen [5]. In this method (Figure 2-b), two filaments $\mathrm{A}$ and $\mathrm{B}$ were twisted together $n$ times. A known force $P_{1}$ was first applied to both filaments. By releasing one end of one of the filaments and charging the other end by a force $P_{2}$, sliding occurred. The coefficient of static friction given by Lindberg and Gralen [5] is as follows:

$$
\mu=\frac{\ln \left(P_{2} / P_{1}\right)}{\pi n \beta}
$$

where $\beta$ is the angle between the two filaments.

El Mogahzy and Gupta [6] have developed another device similar to the one of Lindberg and Gralen, but with a different design in order to facilitate the measurement of friction coefficients between fibers in a liquid medium. 
Their idea was to twist the two fibers by a number of turns, and apply equal forces at one end of each fiber. Increasing the forces on the other ends gave rise to slippage between the fibers.

They derived equation (5) that characterized this type of friction by applying the power relationship between the friction force and the normal force to the movement, demonstrated earlier by Bowden and Tabor [3].

$$
\mu=\alpha\left(P_{1} \beta^{2} / 4 r\right)^{n-1} \quad \text { and } \quad P_{2}=P_{1} \cdot e^{\pi \tau \beta \alpha\left(P_{1} \beta^{2} / 4 r\right)^{n-1}}
$$

where $r$ is the specimen radius,

$\tau$ is the number of twist,

$a$ and $n$ are constants determined from the initial experimental conditions.

To the best of the authors' knowledge, the inter-fiber friction in the transverse direction t-t, as referred to Figure 2, cannot be deduced from measurements in other directions, and this type of inter-fiber friction has not been evaluated yet by any existing device.

However, during transverse strain of multifilament roving, this friction type is prevailing.

Theoretical studies and numerical simulation seem to be very well adapted as far as pointing out clearly and independently the effects of inter fiber or yarn friction on fabric mechanical properties are concerned $[7,8,9]$. Regarding the fiber transverse motion and sliding, Durville et al. [9] showed the importance of the transverse friction on the global transverse compression behavior of multifilament roving. Single polyamide fibers were characterized, then roving with different twist were produced and tested in transverse compression (transverse force as a function of distance between tools). Numerical calculations were also performed with different inter fiber friction coefficients. The simulated transverse compression curves pointed out the significant effect of the friction coefficient on the roving compression behavior. By comparing the curves obtained by experience and by simulation, a friction coefficient of 0.3 was found.

\section{Material and method}

To study the transversal-to-transversal friction, a sliding movement has to be generated between two fibers, according to the directions shown in Figure 1c. Since it is practically very difficult to achieve this movement, it has been replaced by another induced movement, allowing a crossing sliding, as exposed in the right top of Figure 3. An experimental device, 
named Transverse Inter-Fiber Friction Tester 1, has been designed to carry out measurements according to this configuration as shown in Figure 3.

A first fiber, clamped on one end and tensioned by a calibrated mass suspended on the other end, is placed on a rotating and height adjustable plate. This fiber will be called later the 'fixed' fiber. A second fiber is tensioned in the same way and placed on a frame that moves on linear rails. The movement of the frame is operated by a motor whose speed is controlled by a PLC (Programmable Logic Controller). The second fiber will be thereafter called the 'moving' one.

After fiber tensioning and setting the "horizontal" angle AH between them (Figure 4), the plate will be mounted vertically to put the two fibers in contact and adjust the "vertical" angle AV (Figure 4).

Afterwards, the frame, and therefore the ends of the moving fiber will be moved according to Figures 3 and 4 . At the beginning, the friction prevents any relative movement at the contact point. Fibers seem to be 'stuck' together; this point C (Figure 4) moves slowly towards point $\mathrm{C}^{\prime}$. At this point, when the tangential force due to fiber tensioning becomes higher than the fiber-to-fiber frictional force, the slippage occurs abruptly. The slow movement of the 'stuck' monofilaments, as well as the abrupt transition moment -stick-to-slip-, which can be easily observed and recorded on digitals cameras, are perfectly reproducible.

Direct measuring of inter fiber forces, normal and tangential, is very difficult due to their very low levels. On the other hand, the use of any contact sensor perturbs substantially the measure proper. For this reason, the authors have chosen to evaluate these forces by considering the equilibrium state (see Figure 4). 


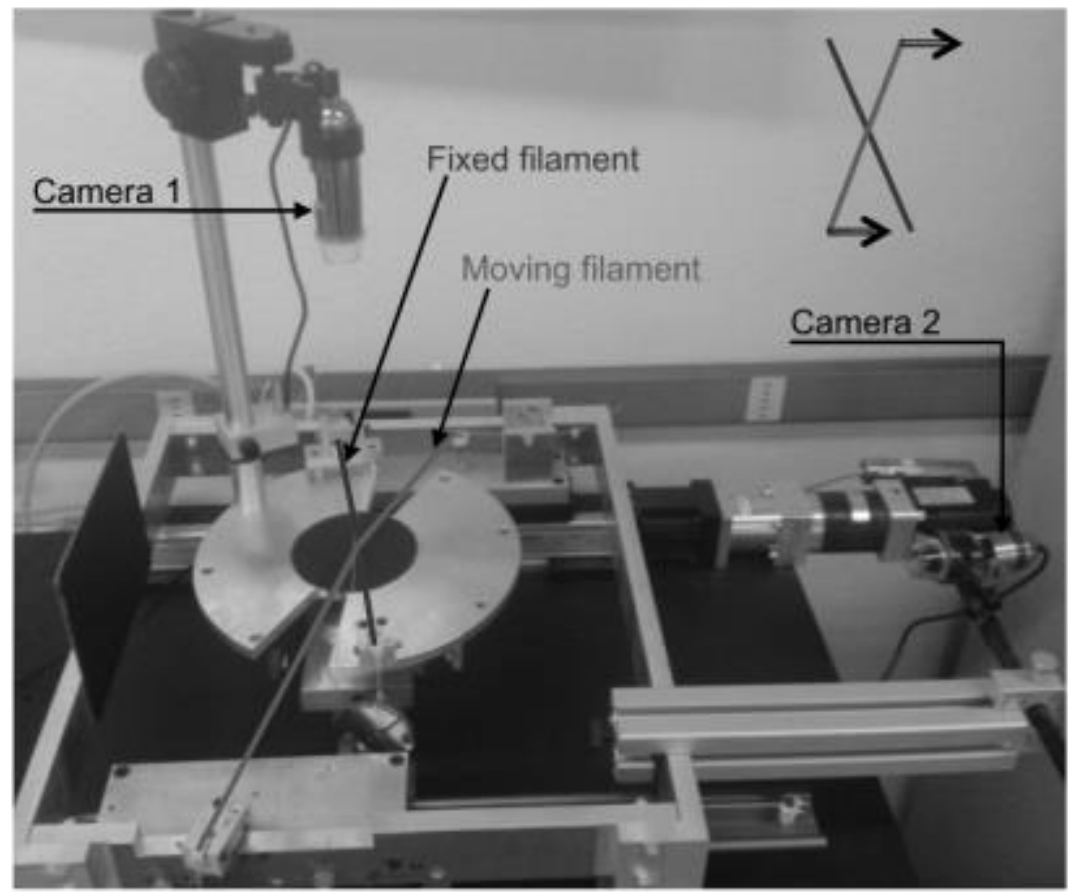

Figure 3. Experimental device: Transverse Inter-Fiber Friction Tester 1

Two digital cameras allow the initial fiber to be positioned and the different angles to be measured just before the slippage takes place, as shown in Figures 3 and 4 .

Dotted lines in Figure 4 show the initial configuration of filaments after being contacted together and before the relative movement takes place, whereas full lines represent the filament position just before slippage occurs. Point $\mathrm{C}$ and $\mathrm{C}^{\prime}$ represent the contact point at these two moments. 

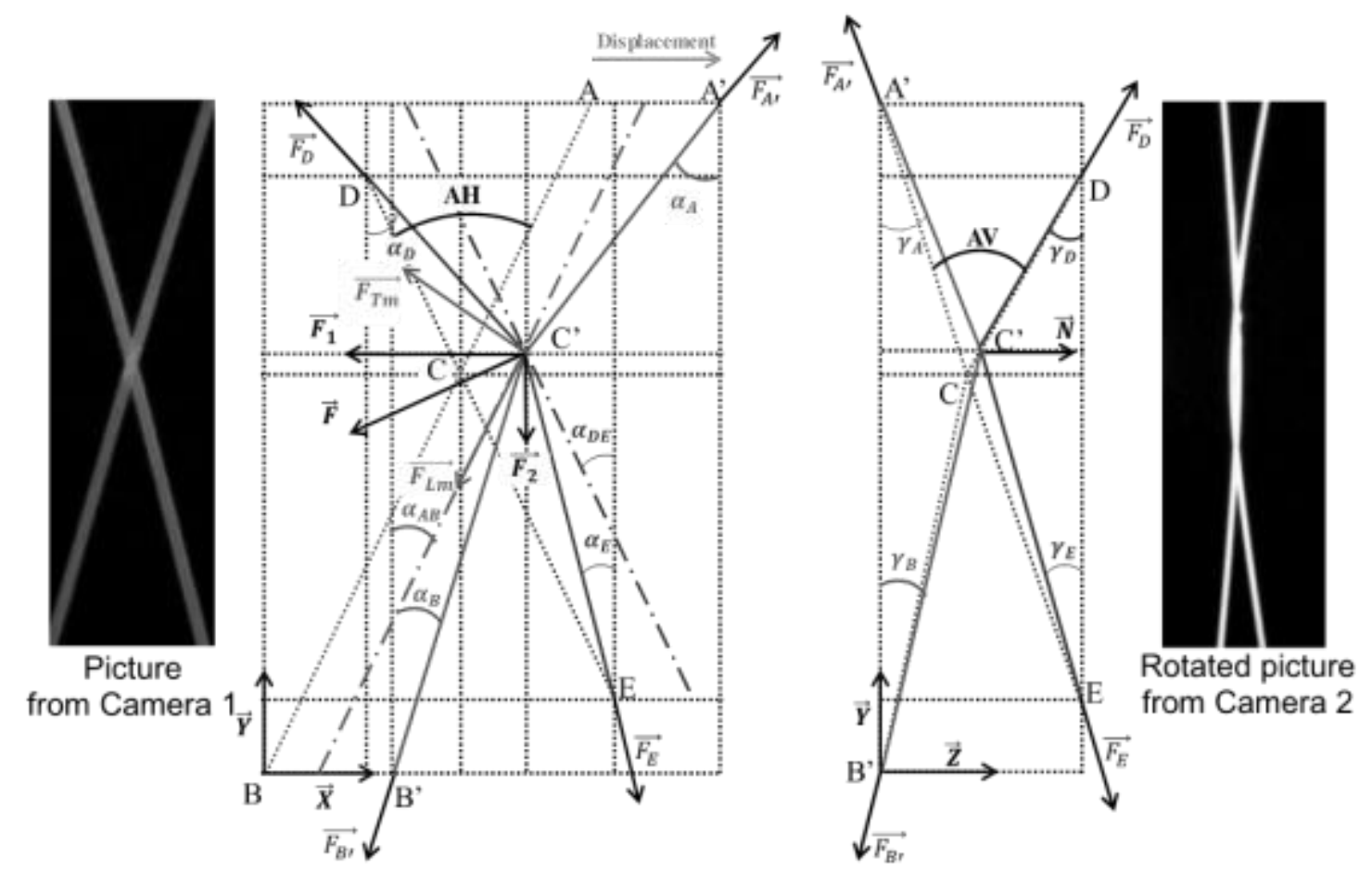

Figure 4. Top and side views of monofilaments in equilibrium during a test in Transverse Inter-Fiber Friction Tester 1; forces applied in moving filament

Two assumptions have been admitted in the following: the contact between the two fibers had been considered as punctual; and torques applied on fibers at the contact point were supposed to be negligible, i.e. their effects would be negligible compared to those of the contact forces. The mechanical equilibrium would depend only on forces applied on the fibers.

Let's note $F_{1}$ and $F_{2}$ the projected forces onto $x$ and $y$ directions, and $N$ the normal force between the two filaments, supposed to be in the $z$ direction. The other involved forces applied to the two filaments are the tensions $F_{A}, F_{B^{\prime}}, F_{D}$ and $F_{E}$. Only the forces $F_{B^{\prime}}$ and $F_{E}$ (fiber tensions), given by two calibrated weights, as well as the different angles $\alpha_{i}$ and $\gamma_{i}, i=$ $A, B, D, E$, given by the two cameras, are known. The angle measurement will be presented here after. In order to calculate the unknown variables: $F_{1}, F_{2}, N, F_{A}$ and $F_{D}$, five equations are necessary. Nine equations are provided by static equilibrium following $x, y$ and $z$ directions: equilibrium of moving filament alone, equilibrium of fixed filament alone, and equilibrium of the two filaments considered together. Considering the projecting angles $\alpha_{i}^{\prime}$ and $\gamma_{i}^{r}, i=A, B, D, E$, as shown in Figure 5 , these equations are as follows (points $\mathrm{A}^{\prime}$ and $\mathrm{B}^{\prime}$ have been noted hereafter as $A$ and $B$ ): 
Equilibrium of moving filament:

$$
\begin{aligned}
& \vec{x}:-F_{1}+F_{A} \sin \alpha_{A} \cos \gamma_{A}^{\prime}+F_{B} \sin \alpha_{B} \cos \gamma_{B}^{\prime}=0 \\
& \vec{y}:-F_{2}+F_{A} \cos \alpha_{A}^{\prime} \cos \gamma_{A}-F_{B} \cos \alpha_{B}^{\prime} \cos \gamma_{B}=0 \\
& \vec{z}: N-F_{A} \sin \gamma_{A}^{\prime}-F_{B} \sin \gamma_{B}^{\prime}=0
\end{aligned}
$$

\section{Equilibrium of fixed filament:}

$$
\begin{aligned}
& \vec{x}: F_{1}-F_{D} \sin \alpha_{D} \cos \gamma_{D}^{\prime}+F_{E} \sin \alpha_{E} \cos \gamma_{E}^{\prime}=0 \\
& \vec{y}: F_{2}+F_{D} \cos \alpha_{D}^{\prime} \cos \gamma_{D}-F_{E} \cos \alpha_{E}^{\prime} \cos \gamma_{E}=0 \\
& \vec{z}:-N+F_{D} \sin \gamma_{D}^{\prime}+F_{E} \sin \gamma_{E}^{\prime}=0
\end{aligned}
$$

\section{Equilibrium of the two filaments together:}

$\vec{x}: F_{A} \sin \alpha_{A} \cos \gamma_{A}^{\prime}+F_{B} \sin \alpha_{B} \cos \gamma_{B}^{\prime}+F_{E} \sin \alpha_{E} \cos \gamma_{E}^{\prime}-F_{D} \sin \alpha_{D} \cos \gamma_{D}^{\prime}=0$

$\vec{y}: F_{A} \cos \alpha_{A}^{\prime} \cos \gamma_{A}-F_{B} \cos \alpha_{B}^{\prime} \cos \gamma_{B}+F_{D} \cos \alpha_{D}^{\prime} \gamma_{D}-F_{E} \cos \alpha_{E}^{\prime} \cos \gamma_{E}=0$

$\vec{z}:-F_{A} \sin \gamma_{A}^{\prime}-F_{B} \sin \gamma_{B}^{\prime}+F_{D} \sin \gamma_{D}^{\prime}+F_{E} \sin \gamma_{E}^{\prime}=0$

where:

$$
\begin{aligned}
& \cos \gamma_{i}^{\prime}=\cos \gamma \frac{1}{\sqrt{1-(\sin \alpha \sin \gamma)^{2}}} \\
& \cos \alpha_{i}^{\prime}=\cos \alpha \frac{1}{\sqrt{1-(\sin \alpha \sin \gamma)^{2}}} \\
& \sin \gamma_{i}^{\prime}=\sin \gamma \frac{\cos \alpha}{\sqrt{1-(\sin \alpha \sin \gamma)^{2}}}
\end{aligned}
$$




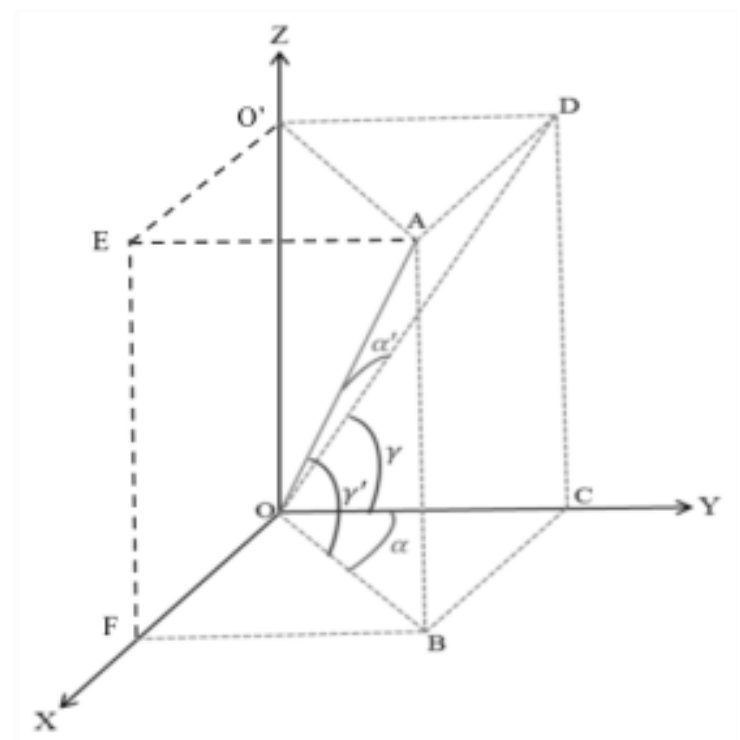

Figure 5. Projection angles used in equations (6) to (8)

Finally, the equilibrium state may be written as follows:

$$
\left(\begin{array}{ccc}
a_{1,1} & \cdots & a_{1,5} \\
\vdots & \ddots & \vdots \\
a_{9,1} & \cdots & a_{9,5}
\end{array}\right)\left(\begin{array}{c}
F_{1} \\
F_{2} \\
N \\
F_{A} \\
F_{D}
\end{array}\right)=\left(\begin{array}{c}
b_{1} \\
b_{2} \\
\vdots \\
b_{9}
\end{array}\right)
$$

The coefficients $a_{m, n}$ and $b_{n}(m=1 . .5, n=1 . .9)$ depend on known angles $(\alpha$ and $\eta$ and forces $F_{B}$ and $F_{E}$. For such a system to have a unique solution $\left(F_{1}, F_{2}, N, F_{A}, F_{D}\right)$, it is necessary that the rank of the matrix $\mathbf{A}$ be different from the rank of the matrix $(\mathbf{A} \mid \mathbf{B})$, which is not the case because of angle measurement errors. It is useful in this case to use the least-squares method and find an approximate solution such as:

$$
\min \|\mathbf{A F}-\mathbf{B}\|^{2}=\min \left[(\mathbf{A F}-\mathbf{B})^{T} \cdot(\mathbf{A F}-\mathbf{B})\right]
$$

This approximate solution was calculated by a simple program developed in Scilab environment [10]. In order to consider longitudinal and transverse frictional forces, the calculated frictional forces $F_{1}$ and $F_{2}$ have been projected on two virtual directions (dotdashed line in Figure 4), the first one given by the angle $\alpha_{A B}$, which is equal to $\left(\alpha_{A}-\alpha_{B}\right) / 2$ and gave the approximated tangent direction to the deflection point $C^{\prime}$ for the moving filament, and the second one was orthogonal to the first, giving this way the longitudinal and transverse frictional forces $F_{L m}$ and $F_{T m}$. The same operation was repeated similarly for the fixed filament by the angle $\alpha_{n F}$, which is equal to $\left(\alpha_{n}-\alpha_{F}\right) / 2$. The longitudinal and 
transverse frictional coefficients have been respectively calculated (see equation [1]) for the two fibers in contact following the formula:

$$
\mu_{l m}=\frac{F_{L m}}{N} \quad \text { and } \quad \mu_{t m}=\frac{F_{T m}}{N}
$$

for the moving filament, and

$$
\mu_{l f}=\frac{F_{L f}}{N} \quad \text { and } \quad \mu_{t f}=\frac{F_{T f}}{N}
$$

for the fixed filament.

In order to validate this new device and the associated mechanical approach, two fiber types have been used in the present study, a polyester one (PES) and a polyamide one (PA). Also, the initial horizontal $\mathrm{AH}$ and vertical $\mathrm{AV}$ angles, as well as the forces $F_{B}$ and $F_{E}$ have been varied, as shown in Table 1 (see also Figure 4)

\begin{tabular}{cc}
\hline Fiber type & $\begin{array}{c}\text { Fiber diameter } \\
(\mu \mathrm{m})\end{array}$ \\
\hline Polyester & 500 \\
\hline Polyamide 6.6 & 230 \\
\hline
\end{tabular}

\begin{tabular}{ccc}
\hline \multicolumn{3}{c}{ Experimental domain } \\
\hline $\mathrm{AH}\left({ }^{\circ}\right)$ & $\mathrm{AV}\left({ }^{\circ}\right)$ & $F_{B}$ and $F_{E}(\mathrm{~N})$ \\
\hline Fixed at 41 & 5.37 to 10.51 & 1 to 2.9 \\
\hline Fixed at 41 & 3.55 to 9.65 & 0.5 to 1.8 \\
\hline
\end{tabular}

Table 1. Material and experimental domain.

The speed of the frame, where the moving filament was mounted, was fixed at of $1 \mathrm{~mm} / \mathrm{s}$ for all tests which seemed to be adapted with the acquisition speed of $30 \mathrm{fps}$ of both cameras. Indeed, the differences between the last and the second last images just before slippage were not important as far as the fiber position changes were concerned.

An image processing was then proceeded to convert them in black and white pictures. Finally, the GIMP (GNU Image Manipulation Program) software was used to evaluate angles. A line was drawn manually so as to make it as parallel as possible to the edge of the fiber.

\section{Results and discussion}

\subsection{Statistical considerations}


It seems interesting, first, to check if the results are significant, i.e. if the results are affected by errors (measurement, environmental conditions, individual and manual process, fiber morphology...). Three replications/individual trial have been carried out for each experimental configuration: the same initial points, same hygrometric conditions, same angles and fibers tensions, but changing the filaments at each trial.

Mean values, standard deviations and regression coefficients have been calculated for each experimental configuration/test. Table 2 summarizes these results of one test using Polyester fiber at experimental conditions: $A V=8.1^{\circ}, A H=41^{\circ}, F B=F_{E}=1 \mathrm{~N}$.

\begin{tabular}{cccccc}
\hline & Trial 1 & Trial 2 & Trial 3 & Mean & St. deviation \\
\hline$\mu_{\mathrm{m}}$ & 0,2191 & 0,2122 & 0,2127 & 0,2146 & 0,003844 \\
\hline$\mu_{\mathrm{tm}}$ & 0,0651 & 0,0693 & 0,0620 & 0,0655 & 0,003694 \\
\hline$\mu_{\mathrm{ff}}$ & 0,2185 & 0,2106 & 0,2111 & 0,2134 & 0,004425 \\
\hline$\mu_{\mathrm{t}}$ & 0,0673 & 0,0645 & 0,0671 & 0,0663 & 0,001564 \\
\hline $\mathrm{R}^{2}$ & 0,931 & 0,935 & 0.933 & & \\
\hline \multicolumn{6}{c}{ coefficient of determination resulting from regression analysis, equation (10) }
\end{tabular}

Table 2. Mean values, standard deviations and regression coefficients of frictional coefficients for one experimental configuration.

The variance of the vector $F$ has also been calculated for each experimental configuration using its three individual trials or replications following the formulae 13.

$$
\operatorname{Var}(\mathbf{F})=\sigma^{2}\left(\mathbf{A}^{T} \mathbf{A}\right)^{-1} \quad \text { with } \quad \sigma^{2}=\frac{1}{n_{e q}-n_{\text {coef }}} \sum_{j=1}^{3} \sum_{i=1}^{9}\left(b_{j i}-\hat{b}_{j i}\right)^{2}
$$

where $n_{\text {eq }}=3 \times 9=27, n_{\text {coef }}=5$,

$$
\mathbf{A}^{T}=\left(\mathbf{A}_{\text {trial 1 }}^{T}, \mathbf{A}_{\text {trial 2 }}^{T}, \mathbf{A}_{\text {trial 3 }}^{T}\right) \quad \text { and } \quad \mathbf{B}^{T}=\left(\mathbf{B}_{\text {trial 1 }}^{T}, \mathbf{B}_{\text {trial 2 }}^{T}, \mathbf{B}_{\text {trial } 3}^{T}\right)
$$

$b_{j i}$ and $\hat{b}_{j i}$ are respectively the experimental value and the calculated one by the model

It is interesting to look at first the coefficients of the matrix $\left(\mathbf{A}^{T} \mathbf{A}\right)^{-1}$ which gives an idea of the covariance between different forces in the vector $F$. Table 3 gives this matrix for the same configuration as in Table 2. Let's remember that the scalars composing the matrix $\left(\mathbf{A}^{T} \mathbf{A}\right)^{-1}$ have no unit. 


\begin{tabular}{lccccc}
\hline & $F_{1}$ & $F_{2}$ & $N$ & $F_{A}$ & $F_{D}$ \\
\hline$F_{1}$ & $\mathbf{0 . 1 7 3 3}$ & & & & \\
\hline$F_{2}$ & 0.0081 & $\mathbf{0 . 5 7 4 5}$ & & & \\
\hline$N$ & 0.0029 & -0.0482 & $\mathbf{0 . 1 7 4 7}$ & & \\
\hline$F_{A}$ & 0.0289 & 0.4358 & -0.0394 & $\mathbf{0 . 5 2 8 5}$ & \\
\hline$F_{D}$ & 0.0114 & -0.4294 & 0.0627 & -0.3966 & $\mathbf{0 . 5 1 3 5}$ \\
\hline
\end{tabular}

Table 3. Example of matrix $\left(A^{T} \mathbf{A}\right)^{-1}$.

It may be observed that covariance coefficients between $F_{1}, F_{2}$ and $N$ are very small compared to the variance coefficients and can be neglected.

From these results, it appeared that the device, as well as the mathematical approach which is attached to it, were not significantly affected by measurement errors. Basically, the coefficient variances are reduced with the values of the matrix $\left(\mathbf{A}^{T} \mathbf{A}\right)^{-1}$ (see equation (13)).

Nevertheless, in order to evaluate the human error in angle measuring, another procedure has been developed. A panel composed of 10 persons had evaluated the angles following the procedure we proposed without any training. Evidently, different persons gave different angle values. The maximal angle difference, which can be considered as an uncertainty, was of $0.1^{\circ}$. The frictional coefficients have been calculated for the angle mean values $\alpha_{i}^{\prime}$ and $\gamma_{i}^{\prime}, i=A, B, D, E$, (equations 9 to 12) as well as for $\alpha_{i}^{\prime} \pm 0.1^{\circ}$ and $\gamma_{i}^{\prime} \pm 1^{\circ}$, giving thus $2^{8}$ $=256$ combinations. Figure 6 visualizes the frictional coefficient distributions as well as the associated quartiles for one experimental configuration at $\mathrm{AV}=9.65^{\circ}, \mathrm{AH}=41^{\circ}, F_{B}=F_{E}=1 \mathrm{~N}$ for the polyamide fiber. Table 4 summarizes the mean values of these coefficients and the respective confidence intervals calculated from the well-known formula:

$$
C I=\frac{\sigma \cdot t}{\sqrt{n}}
$$

where $t$ is the student variable at a confidence level of $95 \%$

$\sigma$ is the standard deviation

$n$ is the total number of the tests. 


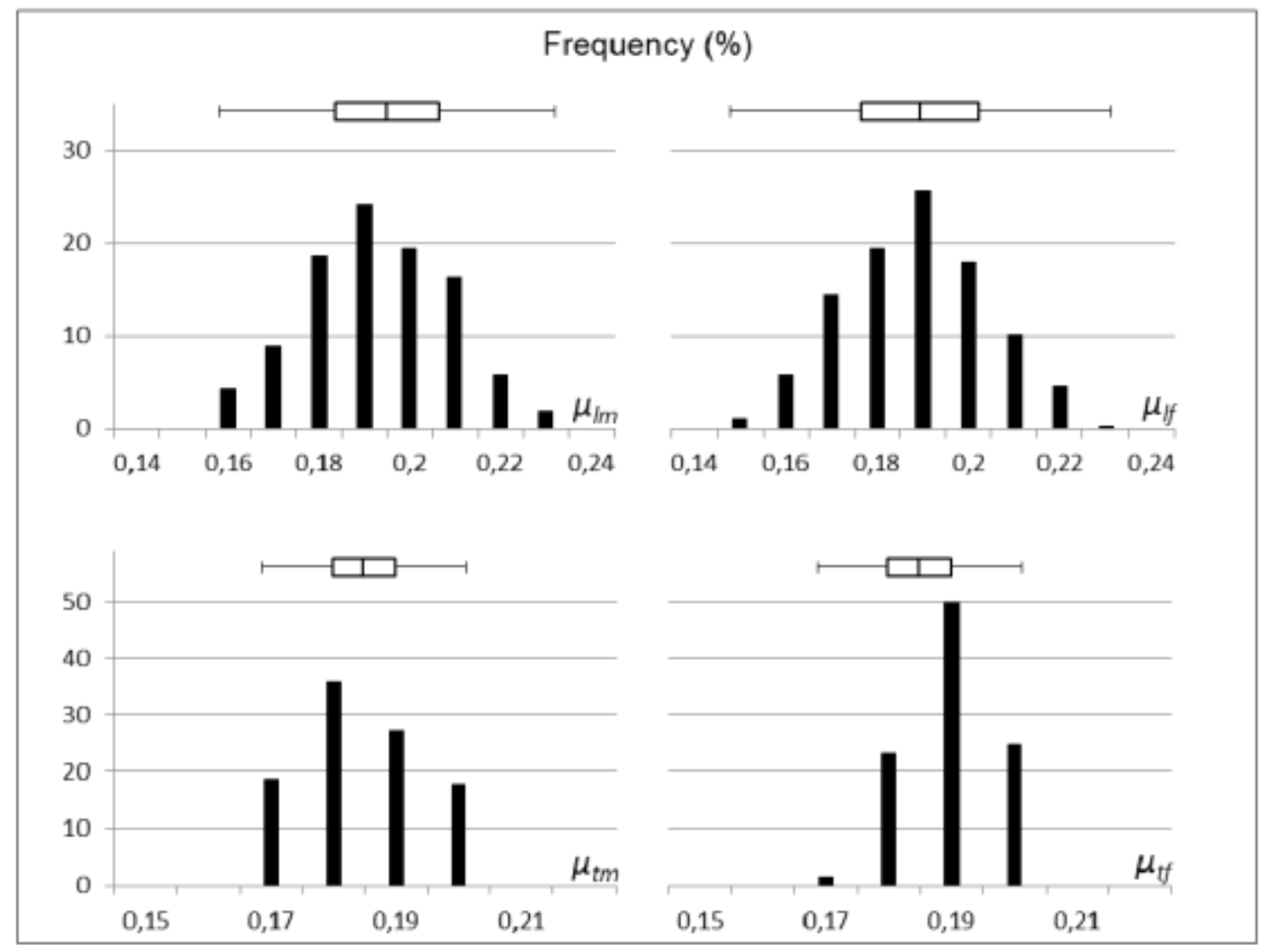

Fig. 6 Examples of the evaluation of the angle error measurement impact on the frictional coefficients

\begin{tabular}{|l|c|c|c|c|}
\hline & $\mu_{\mathrm{lm}}$ & $\mu_{\mathrm{tm}}$ & $\mu_{\mathrm{t}}$ & $\mu_{\mathrm{tt}}$ \\
\hline Mean value & 0,2251 & 0,1836 & 0,2207 & 0,1913 \\
\hline Confidence Interval $\mathrm{Cl}$ & $\pm 0,0019$ & $\pm 0,001$ & \pm 0.0019 & \pm 0.001 \\
\hline
\end{tabular}

Table 4. Confidence Interval of friction coefficients as a function of angle errors

It seems evident from Figure 6 and Table 4 that angle errors do weakly affect the evaluation of the frictional coefficients.

At this point, the topic is to evaluate the confidence intervals of frictional coefficients calculated by the present approach. Let's take the example of $\mu_{\mathrm{lm}}$ and $\mu_{\mathrm{tm}}$ calculated by formula 11: 
The calculation of the variance of these coefficients is not so easy because it implies that $N$ is bounded from below by a known constant. To avoid this problem, the authors propose to evaluate these variances by some simili-confidence intervals, noted here $\mathrm{sCl}$, inspired by engineering uncertainty calculation using partial derivatives (absolute values). According to this method, if a calculated variable $y$ is a function of some measured parameters $x_{i}$, each one measured with a experimental uncertainty $\Delta \mathrm{x}_{\mathrm{i}}$, then the $\mathrm{y}$ uncertainty can be evaluated as follows:

$$
\Delta y=\sum_{1}^{n} \operatorname{abs}\left(\frac{\partial y}{\partial x_{i}}\right) \cdot \Delta x_{i}
$$

Replacing the uncertainties by the simili-confidence intervals, it follows:

$$
\begin{aligned}
& \operatorname{sCI}\left(\mu_{l m}\right)=\frac{\partial}{\partial F_{L m}}\left(\frac{F_{L m}}{N}\right) \cdot \operatorname{CI}\left(F_{L m}\right)+\frac{\partial}{\partial N}\left(\frac{F_{L m}}{N}\right) \cdot \operatorname{CI}(N) \\
& \operatorname{sCI}\left(\mu_{l m}\right)=\frac{1}{N} \cdot \operatorname{CI}\left(F_{L m}\right)+\frac{F_{L m}}{N^{2}} \cdot \operatorname{CI}(N) \\
& \operatorname{sCI}\left(\mu_{t m}\right)=\frac{\partial}{\partial F_{T m}}\left(\frac{F_{T m}}{N}\right) \cdot \operatorname{CI}\left(F_{T m}\right)+\frac{\partial}{\partial N}\left(\frac{F_{T m}}{N}\right) \cdot \operatorname{CI}(N) \\
& \operatorname{sCI}\left(\mu_{l m}\right)=\frac{1}{N} \cdot \operatorname{CI}\left(F_{T m}\right)+\frac{F_{T m}}{N^{2}} \cdot \operatorname{CI}(N)
\end{aligned}
$$

where $C /(N)$ can be calculated by formulae (14), where $n=n_{\text {eq }}=3 \times 9=27$, and $\sigma=[\operatorname{Var}(N)]^{0.5}$ can be calculated by formula (13).

In order to evaluate confidence intervals of $F_{L m}$ and $F_{T m}$ let's remember how these forces were calculated (see Figure 4):

$$
\begin{aligned}
& F_{L m}=F_{1} \sin \alpha_{A B}+F_{2} \cos \alpha_{A B} \\
& F_{T m}=F_{1} \cos \alpha_{A B}-F_{2} \sin \alpha_{A B} \\
& F_{L f}=F_{1} \sin \alpha_{D E}-F_{2} \cos \alpha_{D E} \\
& F_{T f}=F_{1} \cos \alpha_{D E}+F_{2} \sin \alpha_{D E}
\end{aligned}
$$

The confidence intervals of these forces are affected by the variations of forces $F_{1}$ and $F_{2}$ (calculated by the formula 13), as well as the variations of angles $\alpha_{A}$ and $\alpha_{B}$. Using the same principle as previously in equations 15 and 16 , the corresponding simili-confidence intervals will be calculated as follows: 
$\operatorname{sCI}\left(F_{L m}\right)=\frac{\partial F_{L m}}{\partial F_{1}} \cdot \operatorname{CI}\left(F_{1}\right)+\frac{\partial F_{L m}}{\partial F_{2}} \cdot \operatorname{CI}\left(F_{2}\right)+\frac{\partial F_{L m}}{\partial \alpha_{A B}} \cdot \operatorname{CI}\left(\alpha_{A B}\right)$

$\operatorname{sCI}\left(F_{L m}\right)=\sin \alpha_{A B} \cdot C I\left(F_{1}\right)+\cos \alpha_{A B} \cdot C I\left(F_{2}\right)+\left[F_{1} \cos \alpha_{A B}+F_{2} \sin \alpha_{A B}\right] \cdot \operatorname{CI}\left(\alpha_{A B}\right)$

where $C l\left(F_{1}\right)$ and $C l\left(F_{2}\right)$ can be calculated in the same way as $C l(N)$ previously, whereas $C /\left(\alpha_{D E}\right)$ may be estimated at $0.1^{\circ}$, i.e. the maximum angle measurement error.

The mean values and simili-confidence intervals for the experimental combination $A V=9.65^{\circ}$, $\mathrm{AH}=41^{\circ}$ and $F_{B}=F_{E}=1 \mathrm{~N}$, are given in Table 5 .

\begin{tabular}{cccc}
\hline & Mean values & Simili-conf. interval sCl & \\
\hline$\mu_{l m}$ & 0.215 & {$[0.146 ; 0.284]$} & $\mathrm{NS}^{*}$ \\
\hline$\mu_{l t}$ & 0.216 & {$[0.147 ; 0.285]$} & \\
\hline$\mu_{t m}$ & 0.076 & {$[0.025 ; 0.126]$} & $\mathrm{NS}^{*}$ \\
\hline$\mu_{t t}$ & 0.073 & {$[0.023 ; 0.122]$} & \\
\hline$R^{2 *}$ & 0.927 & & \\
\hline
\end{tabular}

not significantly different

" coefficient of determination resulting from regression analysis, equation (10)

\section{Table 5. Frictional coefficient mean values and simili-confidence intervals for one} experimental combination

It results from Table 5 that the simili-confidence intervals of frictional coefficients for moving and fixed filaments have intersecting intervals, which means that there would be no statistical difference between them.

This "no statistical difference" is also globally visualized in Figure 7 where the frictional coefficients of fixed filament (transverse and longitudinal) are plotted versus the ones of the moving filament. 


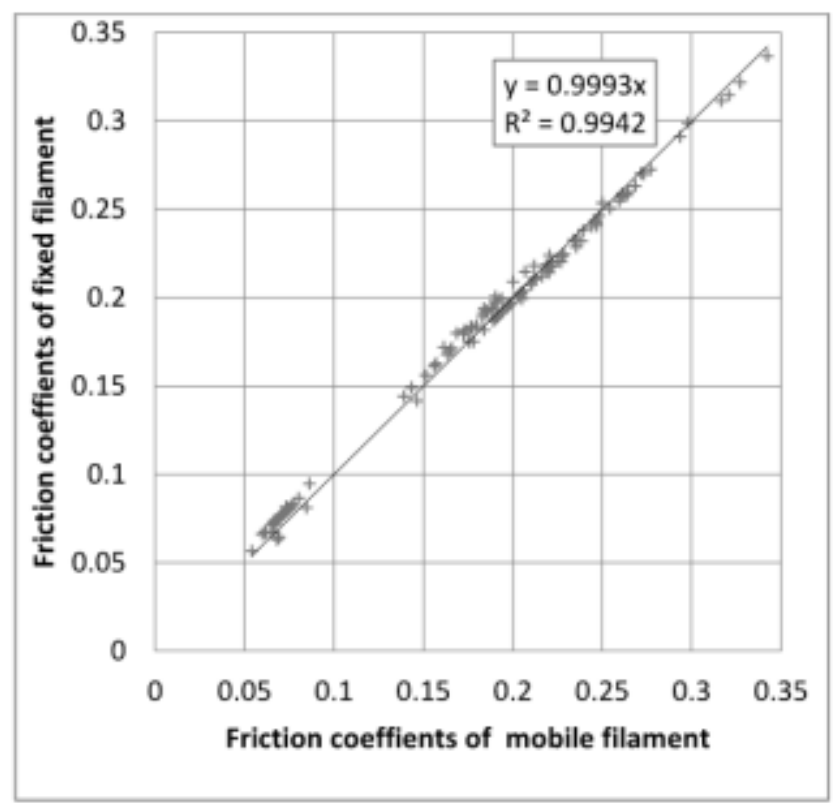

Figure 7. Relation between the friction coefficients of fixed and moving filament

The similitude mentioned here is not a simple experimental observation. On the one hand, there is no physical reason to have frictional behavior differences between the two filaments even if different filaments were used. On the other hand, and logically, from an initial set of 3 independent parameters ( $F_{1}, F_{2}$ and $N$ with very low covariance), it is not possible to obtain by whatever transformation more than 3 independent parameters for example $\mu_{\text {longinudinat }}$ $\mu_{\text {transverse }}$ and $N$.

It will be useful at this point to modify our model (equation 9) by introducing the assumption, verified by the results of Figure 8, i.e. $F_{L m}=F_{L f}$ and $F_{T m}=F_{T h}$ as follows:

$$
\begin{aligned}
& F_{L}=F_{1} \sin \alpha_{A B}+F_{2} \cos \alpha_{A B}=F_{1} \sin \alpha_{D E}-F_{2} \cos \alpha_{D E} \\
& F_{T}=F_{1} \cos \alpha_{A B}-F_{2} \sin \alpha_{A B}=F_{1} \cos \alpha_{D E}+F_{2} \sin \alpha_{D E}
\end{aligned}
$$

which give therefore:

$$
\begin{aligned}
& F_{1}\left(\sin \alpha_{A B}+\sin \alpha_{D E}\right)+F_{2}\left(\cos \alpha_{A B}-\cos \alpha_{D E}\right)-2 F_{L}=0 \\
& F_{1}\left(\cos \alpha_{A B}+\cos \alpha_{D E}\right)+F_{2}\left(-\sin \alpha_{A B}+\sin \alpha_{D E}\right)-2 F_{T}=0
\end{aligned}
$$

With these two additional equations (20), the initial equation (9) is transformed: 


$$
\left(\begin{array}{ccc}
a_{1,1} & \cdots & a_{1,7} \\
\vdots & \ddots & \vdots \\
a_{11,1} & \cdots & a_{11,7}
\end{array}\right)\left(\begin{array}{c}
F_{1} \\
F_{2} \\
N \\
F_{A} \\
F_{D} \\
F_{L} \\
F_{T}
\end{array}\right)=\left(\begin{array}{c}
b_{1} \\
b_{2} \\
\vdots \\
b_{11}
\end{array}\right)
$$

In this case, 7 unknown variables are related by 11 independent equations. The last square method allows to evaluate all variables, where only $F_{L}, F_{T}$, and $N$ will be used to calculate the transverse and longitudinal frictional coefficients.

Table 6 shows the new values of the matrix $\left(\mathbf{A}^{T} \mathbf{A}\right)^{-1}$ for the same experimental conditions as those of Table 3.

\begin{tabular}{lccccccc}
\hline & $F_{1}$ & $F_{2}$ & $N$ & $F_{A}$ & $F_{D}$ & $F_{L}$ & $F_{T}$ \\
\hline$F_{1}$ & $\mathbf{0 , 1 7 3 4}$ & & & & & & \\
\hline$F_{2}$ & 0,0072 & $\mathbf{0 , 5 7 3 2}$ & & & & & \\
\hline$N$ & 0,0030 & $-0,0503$ & $\mathbf{0 , 1 7 5 2}$ & & & & \\
\hline$F_{A}$ & 0,0280 & 0,4345 & $-0,0417$ & $\mathbf{0 , 5 2 7 4}$ & & & \\
\hline$F_{D}$ & 0,0126 & $-0,4277$ & 0,0649 & $-0,3951$ & $\mathbf{0 , 5 1 1 4}$ & & \\
\hline$F_{L}$ & 0,0090 & 0,5419 & $-0,0475$ & 0,4112 & $-0,4042$ & $\mathbf{0 , 5 9 5 8}$ & \\
\hline$F_{T}$ & 0,0031 & 0,1867 & $-0,0164$ & 0,1417 & $-0,1392$ & 0,1765 & $\mathbf{0 , 1 4 4 2}$ \\
\hline
\end{tabular}

Table 6. Example of matrix $\left(A^{\top} A\right)^{-1}$ for the same experimental conditions as those of Table 3 including two additional equations (20)

It is obvious that the variance coefficients of the 5 first variables do not change very much compared to those of Table 3 , and the covariance coefficients between $F_{L}, F_{T}$ and $N$ are very small compared to their variance coefficients.

Now, the calculation procedure is as follows: formula (14) for the variances of forces $F_{L}, F_{T}$ and $N$, where $n_{\text {eq }}-n_{\text {coet }}=3 \times 11-7=26$; formula (14) for the confidence interval $\mathrm{Cl}$ of these forces; and formula (15) and (16) for simili-confidence intervals of frictional coefficients $\mu_{L}$ and $\mu_{T}$, where $F_{L m}$ and $F_{T m}$ are replaced respectively by $F_{L}$ and $F_{T}$.

\subsection{Results analysis}


The calculated transverse and longitudinal frictional coefficients, as well as their corresponding simili-confidence intervals for the two fibers used in the present study are visualized in Figure 8. Note that the values of frictional coefficients obtained by individual trials have also been added in order to point out that they are well grouped presenting thus a practical experimental error lower than the calculated $\mathrm{sCl}$.

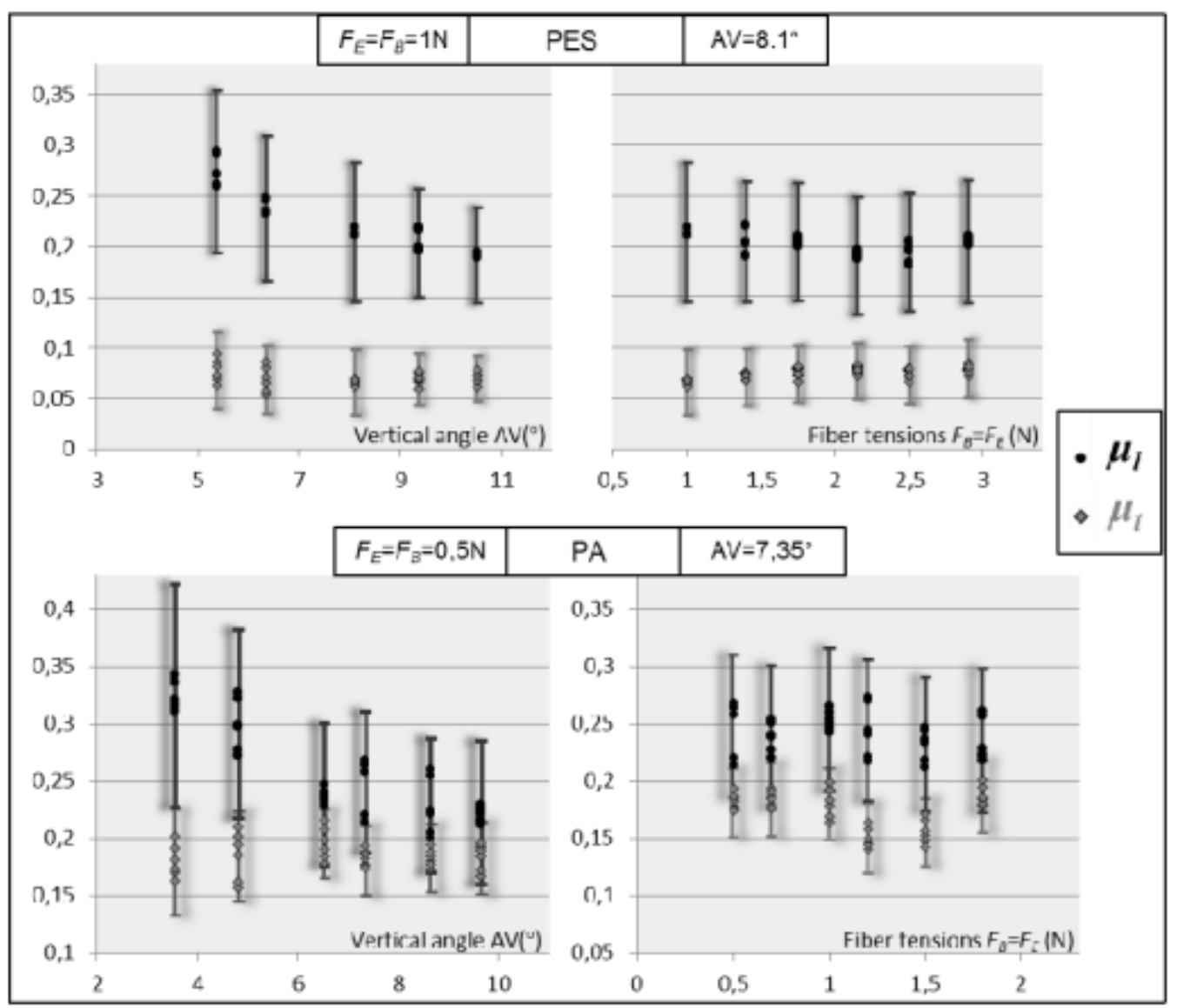

Figure 8. Effect of Fiber tensions and vertical angle on the calculated transverse and longitudinal frictional coefficient

Two major observations may arise from the results of Figure 8. The first one is the fact that the variance are homogeneously or equally distributed in the experimental domain, which is often called homoscedasticity. This statistical quality is very important as far as significant effects have to been discussed and/or pointed out.

The second observation concerns confidence intervals, which are very important to consider the changes of friction coefficients depending on experimental parameters (vertical angle and fiber tension). Let's remember that only the results of 3 trials have been used to calculate $\mathrm{sCl}$ values. It would be interesting to see how the confidence intervals change by 
increasing the number of trials. Figure 9 shows that the confidence intervals decrease strongly with the number of trials. An extrapolation using a power model was performed to estimate the confidence intervals for more than 3 trials, presented also in Figure 9. The two confidence intervals of longitudinal and transverse frictional coefficients would be divided by 2 and 5.2 using respectively 5 and 10 trials, relatively to 3 trial results. A set of 5 complementary trials has been conducted in order to confirm these tendencies for the same experimental conditions as concerned in Figure 9. The confidence interval reduction factor was found to be of 1.95 for both longitudinal and transverse frictional coefficients.

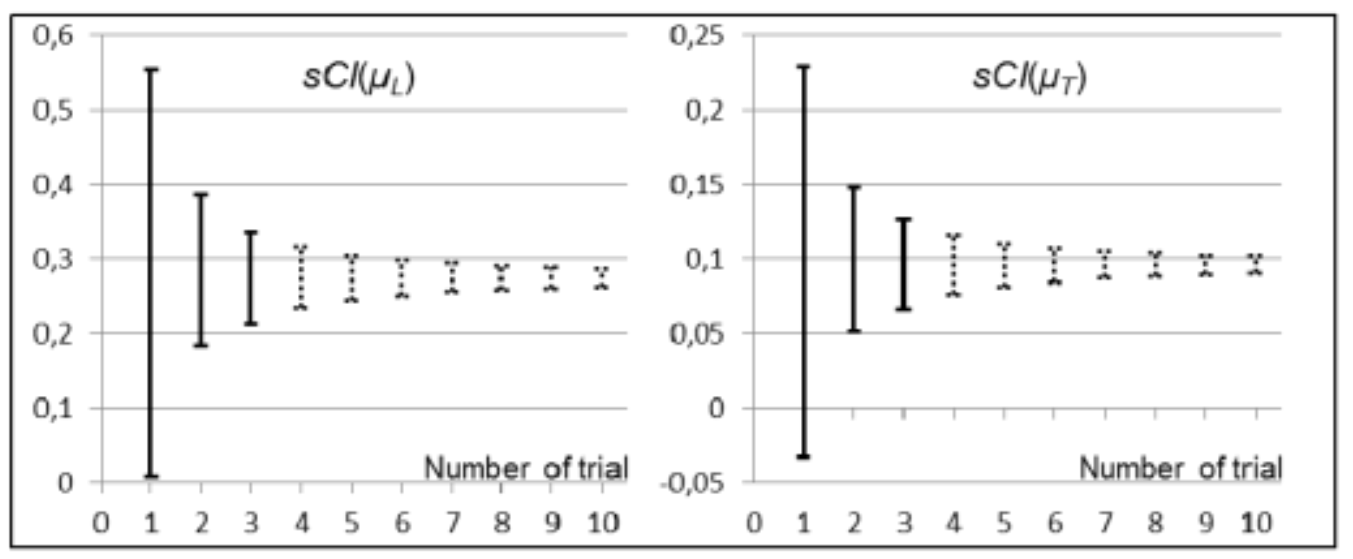

Figure 9. Effect of number of trials on the confidence interval of longitudinal and transverse frictional coefficients, same experimental conditions as in Table 3

As the equality of variance or homoscedasticity has been assumed, it is possible now to discuss the evolution of the frictional coefficients. From Figure 8 , it can be noted first that the frictional coefficients are not affected by fiber tension. As a matter of fact, and as far as the experimental error is not considered, the fiber tension affects quasi proportionally transverse, longitudinal and normal forces (not shown here) giving thus quasi invariable ratios $F / N$. Secondly, the longitudinal frictional coefficients decrease by increasing the vertical angle AV, whereas transverse frictional coefficients seem to be constant independently of experimental factors. Finally, the transverse friction coefficients seem to be lower than the longitudinal one for both considered fibers.

At this point, a question may arise: are there any experimental reference methods to confirm or refute the frictional coefficient calculated in the present study? As it has been mentioned in the background section, nothing is proposed in the scientific literature to evaluate the transverse friction between fibers. On the contrary, the longitudinal friction has been the subject of different studies. 
For this purpose, the authors decided to evaluate the calculated longitudinal frictional coefficient by resorting to the study of El Mogahzy and Gupta [6] described in the background section. A device inspired from their work, shown in Figure 10, was designed and mounted on a servo-hydraulic testing machine.

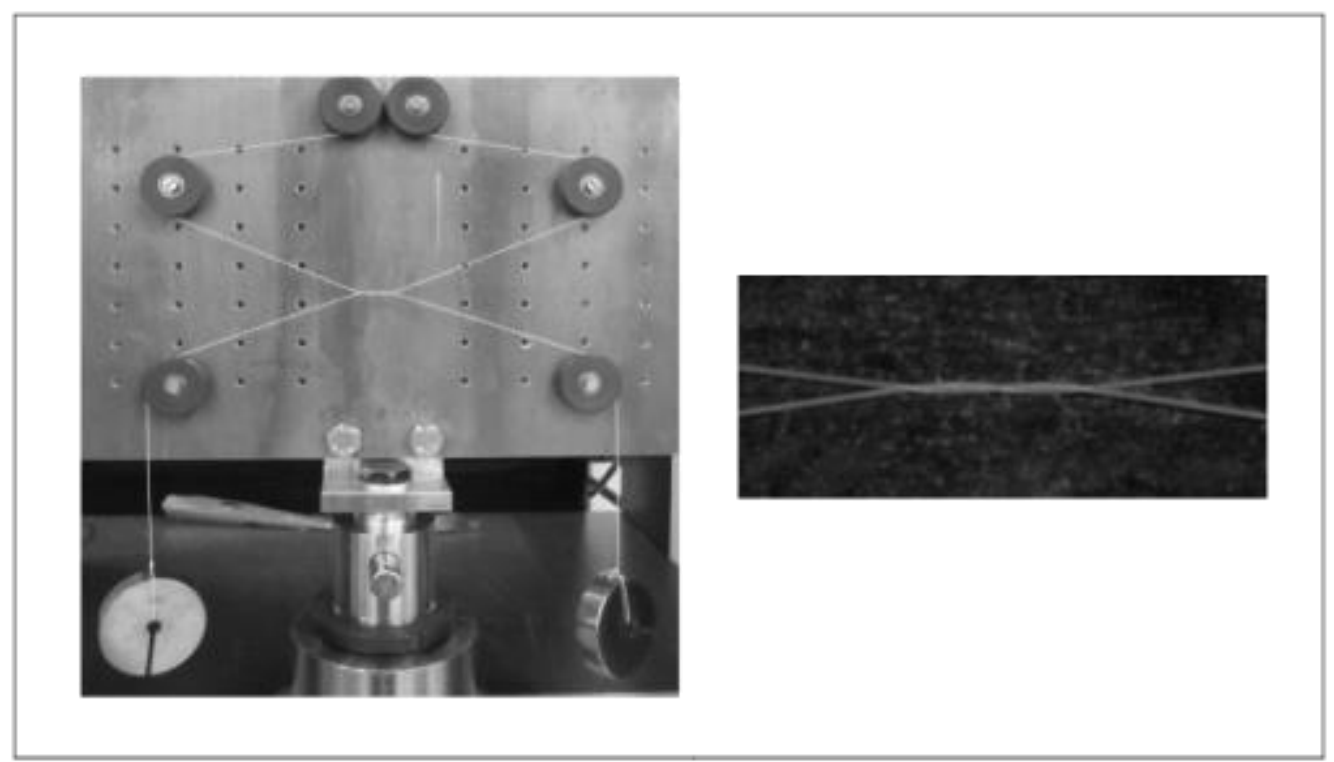

Figure 10. Longitudinal friction device based on EI Mogahzy principle

The tension $P_{1}$ applied to the inferior ends of fibers was maintained constant via two attached calibrated masses. The fibers are twisted once, and then through the pulleys, they are attached to the force sensor of the servo-hydraulic testing machine in order to measure $P_{2}$ force. Different calibrated masses have been used during the experiment. The longitudinal frictional coefficient was calculated by formulae (4).

The measured force $P_{2}$ and the longitudinal frictional coefficient values are shown in Figure 11. The longitudinal frictional coefficient found was of 0.4 for both Polyamide and Polyester fibers, which is superior to those obtained by the method proposed in the present work, respectively from 0.2 to 0.34 and from 0.19 to 0.29 . 


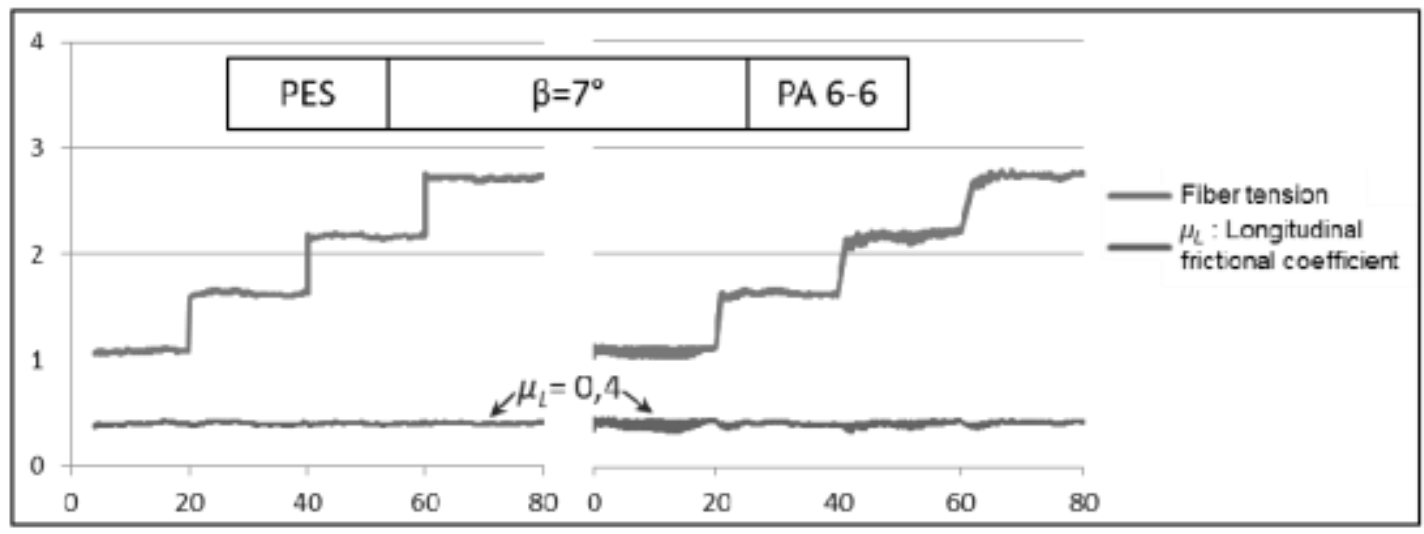

Figure 11. Longitudinal friction results obtained by the experimental device described in Figure 10

At this stage, three observable facts were distinguished: the transverse frictional coefficient evaluated by the present method did not depend on the experimental conditions, particularly the contact angle between fibers; the longitudinal frictional coefficient evaluated by the present method decreased with the contact angle between fibers; the longitudinal frictional coefficient evaluated by an alternative method was more significant than the one evaluated by the present method. There are some inconsistencies between these facts. There is no apparent reason to explain first why transverse frictional coefficient does not depend on experimental conditions whereas the longitudinal one does; and secondly, why the longitudinal frictional coefficient evaluated by another method is different and more significant than the one evaluated by the present method.

To conciliate these results, it will be interesting to consider how the slippage occurs between fibers for the contact fiber configuration presented here. Let's remember that in general, slippage takes place when the external force, tangent to the contact surface, becomes equal or greater than the frictional force $F_{f}=\mu N$. Let's also suppose two independent fiber-to-fiber frictional coefficients, longitudinal one $\mu_{\mathrm{L}}$ and transverse one $\mu_{T}$. During an experimental test described in the present study, external forces $F_{L}$ and $F_{T}$ change continuously. As long as these forces are both respectively lower than their respective limits $\mu_{L} N$ and $\mu_{T} N$, the slip does not occur. When even one of these two external forces reaches or exceeds its respective limit, slippage takes place.

Following this reasoning, it results that in the present experimental device, slippage occurs always in the transverse direction, giving thus 'real' and constant transverse frictional coefficient, whereas the longitudinal force has not yet attained its limit $F_{L}=\mu_{L} N$. The calculated longitudinal frictional coefficient results this way to be variable, underestimated 
and consequently inadequate. Let's remember that the aim of this work was to develop a method for estimating the transverse friction between filaments.

\subsection{Cyclic friction and fiber wear}

It seems interesting at this point to see if the calculated transverse frictional coefficient as well as its confidence interval change during cyclic friction. For this propose, tests have been performed up to 500 cycles. The results (Figure 12) show that the confidence interval does not change, whereas a light decreasing trend of transverse frictional coefficient can be observed. Even if this tendency cannot be affirmed statistically at a confidence level of $95 \%$, the comparison of SEM pictures of virgin fibers and after 500 friction cycles, permit to assume that the light fiber surface wear, caused by friction cycling, gives rise to a light decreasing trend of transverse frictional coefficient.

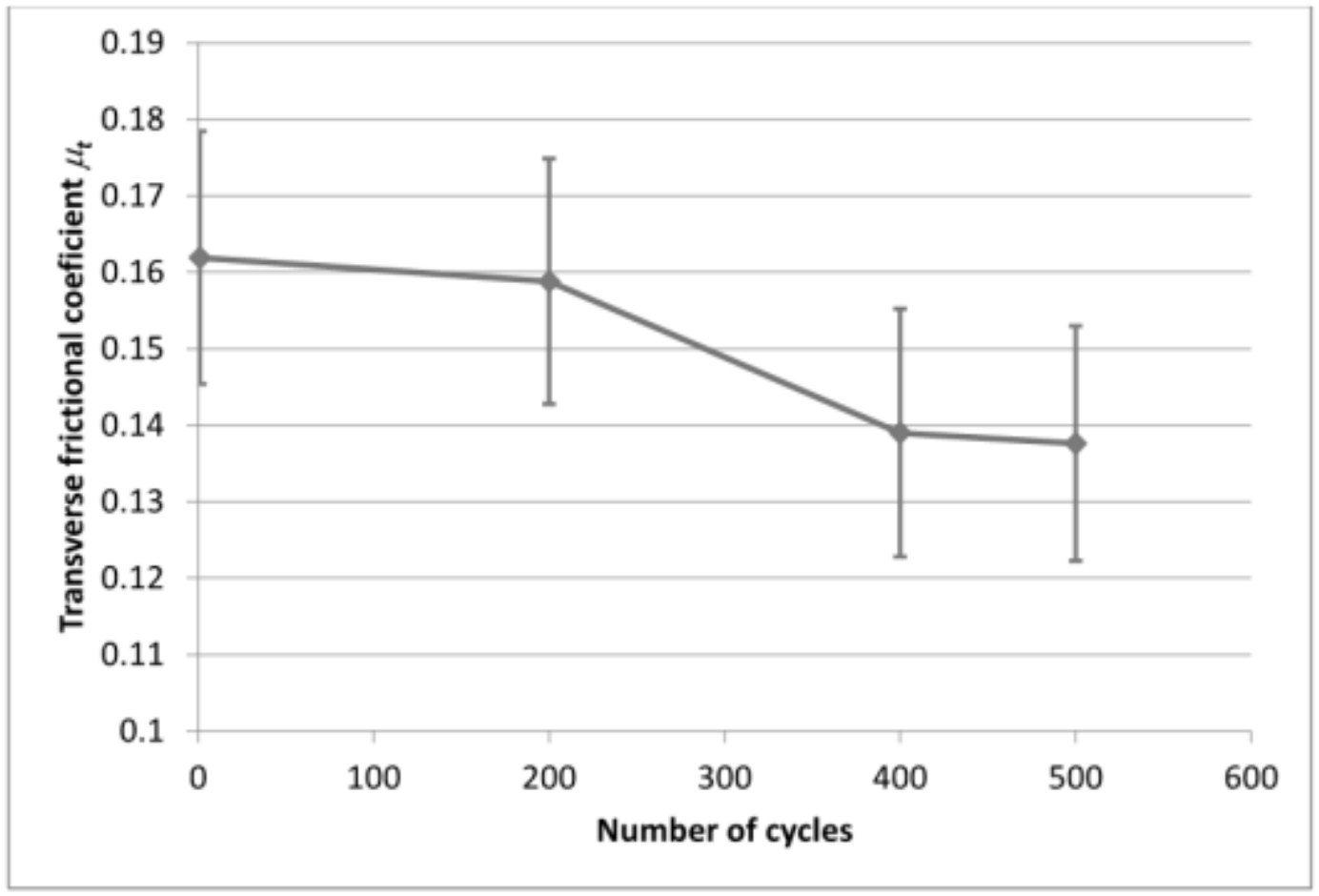

(a) 


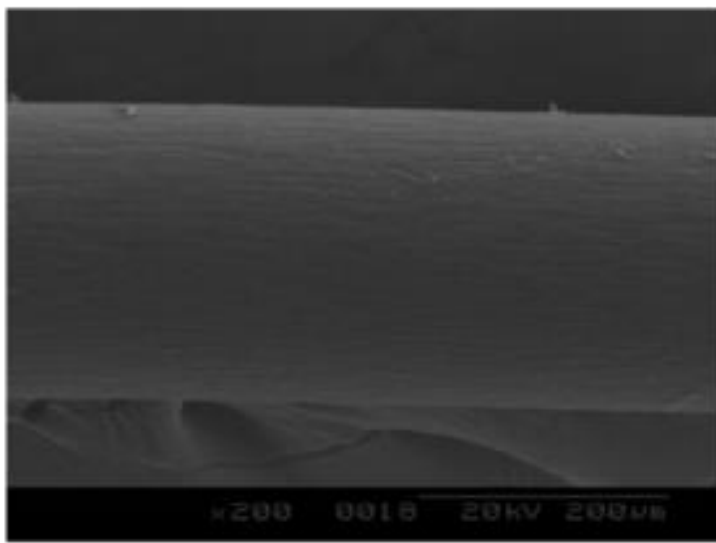

(b)

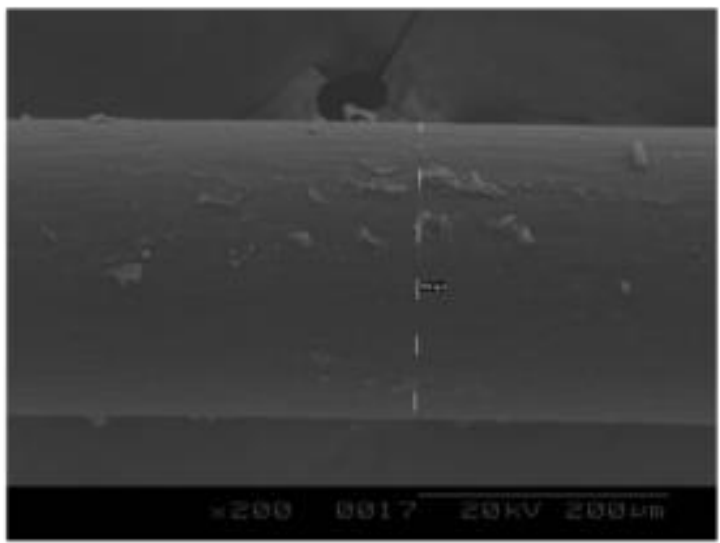

(c)

Figure 12. Friction and wear during cyclic loading, fiber transversal friction coefficient (a), SEM pictures of virgin fiber (b) and after 500 cycles (b)

\section{Conclusion}

Measuring the inter fiber friction, particularly the transverse one, seems to be a difficult topic, first due to the smallness of dimensions and forces involved, and second due to the perturbation caused by the use of possible force or displacement sensors.

A new device and an associated model have been developed in the present study in order to evaluate the transverse inter fiber friction. A relatively simple and original statistical approach has also been developed to evaluate the confidence interval of these coefficients. The first results show a relative stability as far as the error effects and the device configuration parameters are concerned. The transverse friction coefficient seems also to be significantly inferior to the longitudinal one.

It's important to note that this work is a first attempt for measuring the transverse inter fiber friction. Other ideas and/or experimental devices would be very useful to validate the present method.

\section{REFERENCES}

[1] Vinci L, cited in: General Mechanism of Friction, Friction in Textiles, London, Butterworths Scientific Publications, 1959.

[2] Amontons G, Histoire de l'Académie Royale des Sciences avec les Mémoires de Mathématique et de Physique, Paris, Imprimerie Royale, 1699.

[3] Bowden F P and Tabor D, The friction and lubrication of solids, Oxford, Oxford University Press, 1964. 
[4] Howell H G, Inter-fiber friction, J Text I, vol. 42, T521-T533, 1951.

[5] Lindberg $\mathrm{J}$ and Gralén N, Measurement of Friction Between Single Fibers II: Frictional Properties of Wool Fibers Measured by the Fiber-Twist Method, Text Res J, vol. 18, no.5, pp. 287-301, May 1948.

[6] El Mogahzy Y E and Gupta B S, Friction in Fibrous Materials Part II: Experimental Study of the Effects of Structural and Morphological Factors, Text Res J, vol. 63, no.4, pp. 219-230, Apr. 1993.

[7] Kurbak T, Geometrical and mechanical modelings of dry relaxed slack plain knitted fabrics for the benefit of technical textile applications Part II: Mechanical modeling induced by friction, Text Res J, vol. 87, no.7, pp. 853-864, Apr. 2012.

[8] Ben Boubaker B, Haussy B and Ganghoffer J-F, Discrete woven structure model: yarnon-yarn friction, CRAS C. R. Mecanique (2007), doi:10.1016/j.crme.2007.02.006.

[9] Jeguirim S E-G, Fontaine S, Wagner-Kocher C, Moustaghfir N, and Durville D, Transverse compression behavior of polyamide 6.6 rovings: Experimental study, Text Res J, vol. 82, no.1, pp. 77-87, Jan. 2012.

[10]“Documentation / Ressources / Accueil - Scilab.” [Online]. Available: https://www.scilab.org/resources/documentation. [Accessed: 23-May-2016]. 